\title{
Toward a Model Procedure for Social Forecasting
}

\author{
PARIS ARNOPOULOS
}

\begin{abstract}
This article presents the main results of a methodological study on social forecasting. The purpose of the study was to investigate the possibility of a systematic process of making social predictions. The outcome of this research was positive insofar as a "model" procedure was constructed to assess the validity and verify the credibility of tentative forecasts. The article elucidates, in general terms, the principal elements and structural characteristics of the model in a two-dimensional framework (a predictability continuum and a validation sequence) that forms the conceptual matrix of the study. On the basis of this, the operational procedure of the model is given in three phases (probabilism, voluntarism, possibilism) through which any forecast can be tested. The process is illustrated in a flowchart and summarized in a table.
\end{abstract}

\section{Introduction}

Attempting to forecast social phenomena is a most hazardous endeavor. At the core of this difficulty is the human element that infuses all social forecasting with a high degree of unpredictability. In addition, social events depend on so many interacting factors that their evolution becomes a very complex product. Finally, contemporary events unfold with such rapidity as to make it difficult to keep up with them, let alone foresee what follows.

The present state of the social sciences does not possess sufficiently sophisticated methods and concepts to handle so many changing and interacting variables, thus rendering forecasting a very conditional and limited process. For these and other reasons, social forecasts are highly subjective and unreliable. Under the circumstances, perhaps it would have been more prudent not to undertake such forays into the terra incognita of the future.

Yet, for precisely these reasons, the necessity of prediction in public affairs has become increasingly evident. If social systems are to maintain some measure of control over an increasingly complex and accelerating development of the world, they must be able to see ahead, however imperfect such vision can be. This is more so in areas where control is minimal, thus forcing societies to prepare well ahead of any forthcoming eventualities. As social systems become more vulnerable and the repercussions of social problems become more serious, prevention and preparation are the only options for responsible government. In this context of policy planning, social forecasting is the first indispensable step.

Given both the difficulty and the necessity of social forecasting, the question is how to perform this intransigent but unavoidable activity in the best possible way. Naturally,

PARIS ARNOPOULOS teaches political science and public policy at Concordia University in Montreal. 
some kind of forecasting has been going on implicitly throughout human history. But what sufficed in the past is no longer adequate for the future. If one is to go beyond the intuitive speculations of traditional policymaking, one has to search for a more systematic way to supplement human foresight. Whether it is possible to develop a scientific method to study the future remains to be seen; all we can do is keep trying to improve the present techniques.

In any case, the mere process of trying to systematize forecasting regardless of the outcome is instructive in itself and has useful by-products. The search for better ways to look into the future also clarifies the alternatives we have in the present, the objectives we want to pursue, and the effects our actions might have in the longer run. These reasons point to the desirability of forecasting, apart from the necessity of successful forecasts.

It is with these motives that we have undertaken this methodological study of the future; our purpose is to make a first attempt toward a model procedure of generating and assessing social forecasts. To this end, we build a structure and outline a process that will hopefully show how such forecasting is possible. Due to the limitations of an article, we contain ourselves to the most general description consistent with clarity, and we reserve for another time a more detailed explanation.

This article, then, is divided into two main parts, corresponding to the static and dynamic elements of the proposed model. The first part introduces the basic twodimensional framework and the conceptual categories with which we are working. The second part operationalizes these structural elements and thus produces a step-by-step schedule of forecasting activities. In this manner, we present both aspects of the "model" as succinctly as possible.

\section{Structural Characteristics}

To begin with, we construct a forecasting model by outlining its principal elements and the way they are related. These elements may be grouped along two separate dimensions. The first classifies events according to their predictability, and the second subjects tentative forecasts in a sequence of operations that could test their validity. Finally, we combine the two dimensions to show their interrelationship and thus complete the conceptual framework of the model.

\section{PREDICTABILITY CONTINUUM}

Although forecasting is a very difficult and frustrating process, it is by no means impossible. Certain classes of events may be accurately predicted, whereas others seem to be beyond human foreknowledge. Natural phenomena, such as astronomical movements, are well known long in advance of their occurrence; yet, individual behavior-whether of atoms or men-cannot be fathomed even immediately before it happens. There is thus a wide range in the degree of predictability of different events, all the way from the absolute determinism of celestial motion to the total randomness of idiosyncratic behavior.

It appears that there is a spectrum of varying predictability between the two extremes of complete order and chaos. On the one end, there exist the deterministic events of mechanical motion. The bodies in this area follow definite patterns of mathematical precision. Therefore, our ability to predict them is based on knowledge of universal laws and scientific proof. On the other end, there are those unpredictable events of random, unique, or mysterious character. Whether they are by nature inscrutable or it is our present state of ignorance that denies us foreknowledge, such events can nevertheless only be 
foreseen by extrasensory perception or supernatural inspiration. Any attempt to predict them would thus have to be left to the realm of divination and prophesy.

To avoid either an agnostic or a fatalistic position, one has to exclude both extreme determinism and chance as the basis of human action. Instead, we have to assume that man acts as a result of his own "free will" as well as external forces beyond his control. According to this view, man is neither a "plaything of the gods" nor is history "full of sound and fury signifying nothing." Rather, human behavior can be better understood to range somewhere along this hypothetical continuum between randomness and determinism. In this way, we can discern some pattern in human activities and some reason in social events, without expecting to know exactly what is happening or be able to foresee precisely what is coming next.

For purposes of social forecasting, we can safely assume that human activities range somewhere within these limits and thus is somewhat predictable. In this intermediate area of limited determinacy there are various degrees of predictability. On the one hand, there are certain activities with a high degree of predictability, about which we have attained sufficient knowledge to be able to measure the probability of their occurrence. On the other hand, certain other activities are only limited by the nature of what is possible and are thus very difficult to pinpoint as to their future position.

It is within this more limited range of what we consider possible and what we can calculate as probable that we have to concentrate our forecasting efforts. The mixed character of human behavior, which stems both from volition and the laws of nature, place social forecasts in this gray area of predictability. This relative degree of predictability of different types of events may be now summarized in the following continuum:

Chaos

Stochastic-Possibilistic-Volitional-Probabilistic-Deterministic

\section{Random-Incidental-Social-Structural-Mechanical}

Order

In this schema we have coupled social events and volitional behavior at the center of the "chaos-order" range. This centralization focuses our attention in social forecasting and its relation to incidental and structural cvents. Since most human activities fall within these three middle categories, we have to explain this approximate coincidence and relativity of incident-possibilistic, social-volitional, and structure-probabilistic events.

The first type of event is closest to the random end of the range and hence is relatively the least predictable group. The events of this group involve individual or particular activities of such idiosyncratic nature as to make them almost impossible to predict. Here we encounter the human element in its most unpredictable form, so we can only speculate about its future. One can do so by using personal experience, psychological insight, or gifted imagination. This is a most frustrating group because it includes historical surprises, cultural revolutions, innovative breakthroughs, and other dramatic events that bring about the occurrence of the unexpected and the nonoccurrence of the expected.

On the contrary, the third type is closest to the deterministic end and thus is the most predictable group. It contains rather structural or technical activities that have a high probability of projection. Technological forecasting (i.e., all weapons systems of the next decade have already been invented); continuing trends (i.e., the population of the world will keep increasing to about six billion by the end of this century); and institutionalized routines (i.e., the U.N. General Assembly will meet in September every year for the 
foreseeable future). There are many events of this type that can be predicted with a high degree of confidence and it is on the basis of such predictability that the general stability of social systems is maintained.

Between these two types of events lies social forecasting. Collective behavior is not so predictable as structured patterns, but neither is it so unpredictable as particular incidents. In this intermediate area we have cerlain social-science hypotheses, historical analogies, and purposive activities, so that we could make some cautious forecasts. Since the events in this group depend as much on human volition as on external constraints, prediction can only be conditional and provisional, such as: political forecasts (if trends continue, the governing party will loose the elections), logitudinal comparisons (history repeats itself, but not quite), and proposed policies (the 5-year plan intends to increase production by $5 \%$ per annum). Evidently, forecasts of this type combine both objective and subjective considerations into some credible probability. It is in view of improving our capacity to make such forecasts that we should increase our knowledge about the nature of these events.

\section{VALIDATION SEQUENCE}

The other dimension of our forecasting model building deals with a sequence of steps by which tentative forecasts can be systematically validated. This dimension, as the previous one, could be imagined as a linear construct with various salient points along it. Unlike the predictability continuum, however, this line represents a sequence of events that, if successfully concluded, should convert a subjective premonition into an objective forecast. Of course, this transformation only validates the forecast; it by no means guarantees its fulfillment.

Forecasts are conditional propositions about the future. As such, they are hypothetical (if . .., then ...) statements regarding a course of events that might happen beyond the present time. Since we have rejected the absolute inevitability of social events we have to accept our limited capacity to predict human actions. All we can do, therefore, is to ensure that statements about the future undergo as rigorous a testing as possible before they are accepted as "scientific."

What we are looking for is a systematic way of judging whether a claimed forecast is valid to the best of our knowledge. This judgment should be based on whether the claim can pass certain tests that will show the rigor of its construction. We are here proposing that such validation sequence must contain certain essential elements, ranged along the following schedule:

Input $-\frac{\text { Basic }}{\text { premises }} \rightarrow-\frac{\text { Documentary }}{\text { evidence }} \rightarrow-\frac{\text { Predictability }}{\text { criteria }} \rightarrow--\frac{\text { Testing }}{\text { calculus }} \rightarrow$ Output

Since we explain the operational procedures of this sequence later on in the article, we limit ourselves here to describing the nature of these four steps in simplified form.

The first ingredient in any scientific methodology is to assume explicitly the basic premises on which the whole edifice of the reigning paradigm rests. Geometrical axioms, mathematical postulates, and elementary definitions all fall within this area. Without such assumptions, one cannot build the necessary theories to explain "reality." If forecasting is to become scientific, it has to accept these premises and develop certain corollaries for its specific requirements. 
The second requirement is epistemological and concerns the sources of evidence that constitute the basis for forecasting. Since empirical evidence exists only for the past and present, the study of the future must contend itself with projections and inferences from this evidence. It is this inherent difficulty that makes the rules of inclusion and manipulation of evidence so crucial in forecasting. Only after there is some success in this enterprise will we be able to accumulate, store, select, and recall the proper information leading to valid forecasts.

Once we have adequate information and relevant theories, we should be able to make correct interpretations of the evidence relative to the future. This is the third step of our sequence, where a judgment has to be made as to the degree of predictability of the proposition in question. Here one has to determine whether there is sufficient indication of the existence of data and concepts to justify scientific forecasting.

It is at this point where we encounter the predictability continum that we explained previously. The question of theoretical and empirical adequacy cannot be answered absolutely one way or another. In practice, there is always partial information and conditional theories, so the answer has to be somewhere between the ideal and the minimal requirements for an acceptable forecast. Social forecasting, as we mentioned, falls in the middle of this continuum; therefore, it admits of some degree of predictability.

Assuming a correct decision on this matter, the final step would be to perform the operations necessary for the determination of the predictive parameters. That is to say, the conditions under which the forecast will be valid, the probability of its materialization, and the time span covered. If these variables could be measured within an acceptable range of error, we may speak of a valid forecast. Here again, validity can only be relative, depending on the degree to which a tentative forecast passes or fails these tests. In any case, the outcome of this process should tell us something about the credibility of a proposition concerning the future.

\section{CONCEPTUAL MATRIX}

We are now ready to combine the two dimensions outlined above, into an interrelated framework. By placing the two linear schemas at right angles to each other, we can construct a two-dimensional plane that shows the relationship between validity and predictability in various cross-cutting stages. Since the first dimension has three salient points and the second dimension has four main steps, their orthogonal combination results in a basic $3 \times 4$ matrix. To this, we have added the input and output stages, along with the generic terms, to construct Table 1 .

Table 1 summarizes the points we have already made but then goes on to illustrate the characteristics of each of the resulting 12 central combinations. The terminology in these boxes is elucidated in the operational procedures of the next section; all we do now is to explain the basic structure of the matrix and the rationale of its divisions.

The first column is in essence the validation sequence as it was outlined in the previous section; therefore, no further explanation is necessary at this point. The next three columns represent the three relative classes of the predictability continuum, from the most to the least predictable type. Thus, in the first place, we have the realm of structural events that are most prone to prediction. This is the closest we can approach scientific forecasting, as is evident in some technological forecasts.

At the other end, we have those incidental—not to say accidental - events that are least prone to systematic prediction. For them, we have to rely on subjective criteria and educated guesswork. These two categories form the boundaries of social forecasting and 
TABLE 1

Validity-Predictability Matrix

\begin{tabular}{|c|c|c|c|c|}
\hline $\begin{array}{l}\text { CLASS } \\
\text { Scope } \\
\text { Field } \\
\text { Range }\end{array}$ & $\begin{array}{c}\text { STRUCTURAL } \\
\text { Nature } \\
\text { Technology } \\
\text { Short }\end{array}$ & $\begin{array}{l}\text { SOCIAL } \\
\text { Culture } \\
\text { History } \\
\text { Medium }\end{array}$ & $\begin{array}{c}\text { INCIDENTAL } \\
\text { Personality } \\
\text { Literature } \\
\text { Long }\end{array}$ & INPUT \\
\hline PARADIGM & THEORY & HYPOTHESIS & DOCTRINF & AXIOM \\
\hline Premise & Postulate & Concept & Belief & \\
\hline Source & Knowledge & Assumption & Experience & \\
\hline Context & Principle & Generality & Ideology & \\
\hline EVIDENCE & DATA & PLAN & OPINION & REFERENCE \\
\hline Information & Quantity & Intention & Impression & \\
\hline Index & Statistic & Precedent & Speculation & \\
\hline Document & Function & Policy & Authority & \\
\hline CRITERION & PROBABILITY & VOLITION & POSSIBILITY & JUNCTURE \\
\hline Realm & Science & State-craft & Art & \\
\hline Selection & Empirical & Behavioural & Idiosyncratic & \\
\hline Decision & Rationality & Realism & Credibility & \\
\hline METHOD & CALCULATION & ANALYSIS & CONSENSUS & OPERATION \\
\hline Test & Experiment & Analogy & Simulation & \\
\hline Process & Induction & Inference & Imagination & \\
\hline Measurement & Correlation & Comparison & Survey-poll & \\
\hline RESULT & PROGNOSIS & PREDICTION & PREVISION & OUTPUT \\
\hline Product & Projection & Expectation & Scenario & \\
\hline Proof & Explanation & Elucidation & Conjecture & \\
\hline Goal & Extrapolation & Prescription & Revelation & \\
\hline
\end{tabular}

define its scope. It is, therefore, necessary that social forecasting utilize a range of techniques with varying degree of scientific rigor, in order to arrive at some optimal forecasting mixture.

As Table 1 shows, this range varies from the explicit theories of the natural sciences to the implicit beliefs of human experience, from the hard quantifiable data of statistics to the soft qualitative personal impressions, and from mathematical calculation of probabilities to narrative speculations of alternative scenarios. A careful selection of these different tools for different occasions should maximize our forecasting capabilities.

The last column of the matrix in Table 1 illustrates a series of symbols that correspond to the various steps of the validation procedure. As we see in the next section, these symbols are used to construct the operational model for the forecasting assessment. Therefore, they serve to frame the different concepts presented in the other columns transposed to the forthcoming model.

Finally, Table $I$ and the preceding terminology should be read in the context of the article and along with the explanations given in the text. For that reason, the terse labels of the matrix in Table 1 should be interpreted in a flexible rather than literal manner. As in the case of all taxonomies, our categories and their characteristics are analytic and somewhat arbitrary. Reality does not fit neatly in the compartments of our minds. The traits we have attributed to each box spill over to the neighboring ones and overlap in grey areas, rather than thin lines. The usefulness of the matrix, then, is to highlight at a glance the salient points of the complex elements and relationships involved in the forecasting process. 


\section{Operational Procedures}

Now that we have completed assembling the elements of the proposed model and placing them in relative perspective, we can attempt to operationalize the resulting structure into a forecasting assessment procedure. For this purpose, we have rearranged the elements into a stylized chart (Fig. 1). Figure 1 shows the dynamic relationships between the elements and thus converts the validity-predictability matrix into the forecasting process.

In order to transform a static matrix into a flowchart we have utilized arrows that connect the elements in temporal sequence. These arrows indicate both the direction of the process and the quality of the connection (i.e., solid line $=$ strong; dash $=$ medium; dotted = weak). The overall process is divided into three phases, corresponding to the three middle columns of the matrix. Each phase is subdivided into four main steps, corresponding to the four central rows of the matrix. In order to explain Fig. 1 we deal with each phase in turn in the three following sections.

\section{PROBABILISM PHASE}

The forecasting process may be initiated by either posing a question about the future (e.g., "What will the world be like in the next generation?") or making a tentative forecast (e.g., "The next generation will live much better than the present!'”). Either way, we want to determine to what extent we can answer the question or confirm the proposition by some objective method. It is to this end that we process this subject matter through the flowchart (Fig. 1).

We begin at the upper-left corner of Fig. 1 with as specific a question as possible (e.g., "What will be the population of the world in the year 2000?") or a particular intuition about an answer (e.g., "The world's population in the year 2000 will be approx-

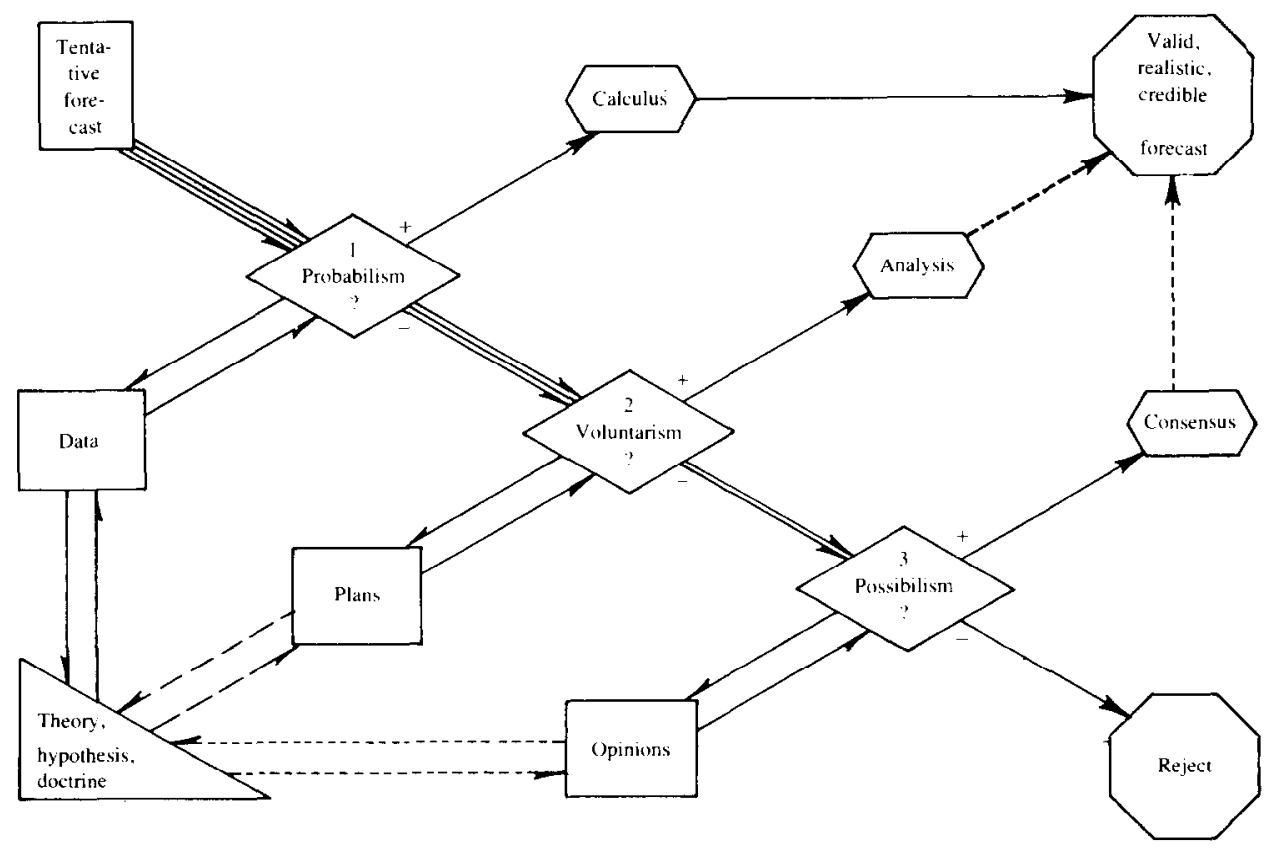

Fig. 1. Forecast-assessment flowchart. 
imately six billion."'). To find out something about this future situation, we should know whether the proposed event is structural, volitional, or incidental so that we can determine how well we can predict such a thing.

To do so, we first ask whether we can find the probability of various alternative futures occurring under certain conditions. Since we have rejected the certainty of any social forecast, the best we can do is to see how highly probable any given forecast is (e.g., "Can the probability of a particular number of people living in the future be ascertained?''). This, in effect, is the question posed in the first juncture of the flowchart (Fig. 1).

In order to answer this question we must determine whether there is adequate evidence pointing toward any particular direction more than in others for the future evolution. Since the only evidence available concerns the past, one necessarily has to rely on historical data. If the research uncovers enough information of high quality, it may be possible to extrapolate it into the future. In some cases, there is good statistical data going back in time to allow for the establishment of definite trends that can then be projected in time. If there are no adequate data, our question must be answered in the negative, and the probabilism test fails.

Hard data, however, are insufficient to answer the question positively. Also necessary is the existence of adequate theory to interpret the data. One must, therefore, make sure that there is proper substantive and procedural knowledge by which the given facts can be explained and manipulated. Without such backing, raw data can turn out naive projections and simplistic forecasts.

Unfortunately, good theories are more difficult to find in the social sciences than is reliable information. (Although many countries keep accurate demographic statistics, it is still difficult to establish trends, given the changing patterns of natality, mortality, and migration.) In its present state, social science has only developed quasitheories or very limited generalizations, so that projections of data can only be done cautiously.

Nevertheless, in few areas (i.e., demography), there are adequate data and techniques to warrant a positive answer to the probabilism question. In this case, one could determine what is the probability that a given forecast would occur. This would require that the necessary data and the theoretical premises be brought together in calculating as closely as possible the chances that different alternatives could develop in a certain time period (e.g. " "There is a $90 \%$ chance that the population of the world in the year 2000 will range between five and seven billion.").

If such calculations can be made, the resulting forecast may be considered valid. Of course, only time would prove it correct or not, but for purposes of the present it would have to be accepted until proven wrong. Such properly formulated forecasts seldom come by in the social sciences. Lack of knowledge or information make forecasting calculations meaningless, if not dangerous. In these cases, it would be better not to claim scientific rigor, or to supplement a questionable probability with other methods.

Assuming that in most cases of social forecasting, the preceding validation procedure would be either impossible or doubtful, one would have to apply less exacting standards for forecast assessment. In terms of Fig. 1, this would mean having to take the negative (-) path out of the first criterion, leading to the next phase.

\section{VOI IJNTARISM PHASF.}

In this testing sequence we replace most of the quantitative procedures of the first phase by qualitative judgments. In the absence or paucity of the former, the next best thing 
is to try the latter. In this case the verification criterion will no longer be probabilistic. Instead, we ask similar questions, but in a voluntaristic context.

We have assumed that the actions of social groups stem not only from their objective capabilities, but from their subjective will. The type of events considered in this category, therefore, result to a large extent from the actors' conception of the past, perception of the present, and intention of the future. If we are to forecast social activities, we must take into account not only historical traditions and contemporary images, but also future plans and aims.

For that reason, the central question of this sequence is whether we can find out what the dominant actors of the social system plan to do? (e.g., to determine if the North-South gap will keep increasing in the next decade, one should understand the policies of the great powers in this respect, among other things). It is to this end that the first step becomes research into government policies and intentions.

Here, as in the case of statistics, the availability of documents leaves a lot to be desired. Government secrecy and human indecision both make accurate plans difficult to come by. Yet, there is considerable evidence of what social actors intend to do. There are many indications of future action (private interviews, public speeches, 5-year plans, laws, and treaties) that can be used as an aid to forecasting.

What people say they will do, of course, is not necessarily what they end up doing. There is thus a discrepancy between intentions and actions, which the sophisticated forecaster must take into account. To do so, he must have some psychosocial hypotheses to explain such discrepancies. One must have some ideas on human nature, national character, and cultural traits in order to make valid inferences from the documentary evidence.

Unfortunately, here too, our knowledge of man and society is not so advanced as to allow us accurate prediction. Moreover, even if it could, the subject of the prediction could, once he becomes aware of it, change his mind and act in such way as to make our forecast a self-fulfilling or self-denying prophecy. These inherent limitations render social forecasting unavoidably provisional. Our assumptions and generalizations can only be made conditional to many factors. For this reason, the phrase "other things being equal" is the refrain of social scientists.

Nevertheless, given all these constraints, it is still possible to attempt a systematic policy analysis that takes into account as many of these variables as possible. There are certain techniques (content analysis, cost-benefit calculation, relevance trees, crossimpact tabulation, etc.) of analogy and comparison that can serve as a good basis of volitional forecasting. An experienced political analyst with good information sources could make fairly accurate predictions of realistic freedom of action within which the future will most likely unfold.

Since neither the first nor the second criterion alone is sufficiently reliable for social forecasting, in combination they improve our chances of prediction. Thus using whatever hard data one can get on what social systems are capable of doing, as well as whatever qualitative evidence is available on what powerful actors are intending to do, a forecaster will cover both bases of human action and thus maximize his results. As an example, the North-South gap has been reproduced by increasingly sophisticated econometric models of the Club of Rome and the United Nations. These complex models have determined the various conditions under which the gap will increase or decrease. These conditions include both natural constraints and government policies, so several permutations of these give us the alternative futures of the world in this matter. (All things considered, it is rather unlikely that the gap will be closed in the foreseeable future.) 
In any case, alternative forecasts serve to point out the options open to us, so that we can choose to bring about the most desirable or the lesser evil future. By revealing the different directions we can take, these forecasts not only set the range of our expectations, but prescribe the priorities of political action. Thus, they encompass the scope of what people are willing and able to do to determine their future.

At this point, however, we must move on to the next and last criterion of social forecasting concerning the credibility of what is possible in the future. If the first two phases were properly fulfilled, we may not have necded the third onc. But for all practical purposes, we would require additional confirmation of our forecasts, so we use the following criterion for that.

\section{POSSIBILISM PHASE}

This ultimate test can be used either to complement the previous ones or to forecast those events that cannot be validated by calculation and analysis. This area includes controversial cases that resist methodical solutions and involves inscrutable elements beyond rational consideration. It thus approaches the limits of our consciousness and enters the realm of inspiration. The only criterion left is that of credibility: whether the best minds available believe that a given forecast is feasible.

In order to answer this question one has to resort to the prevailing opinions of the present. In a sense, subjective judgment as well as objective knowledge are involved in policy analysis and even the interpretation of statistical data. But in this phase, subjectivity predominates over the other forms of evidence. Under the circumstances, all one can do is render such subjective procedure more systematic by tapping the collective wisdom, enlightened ideas and the imaginative spirit of the world. The educated guesses produced by such process should give us some glimpse into the future.

One should be careful to note that behind the opinions of people lie cultural beliefs, political ideologies, and historical doctrines. Personal opinions are shaped by these traditional forces, even in the best of minds. On this basis, people often foresee only what they want to see and confuse aspirations with expectations. We should, therefore, make allowances for these factors and separate the different elements of opinions about the future.

Unlike the dearth of information that exists in the previous two areas, in this one we can easily find a plethora of opinions. It is, therefore, crucial how we select and treat these opinions. The test utilized for this criterion may emphasize either individual creativity or group consensus. In the former, a single mind synthesizes various ideas and facts into a scenario about the future or weaves variations on a theme of possible futures. In the latter, the collective expertise of many people is harnessed through various interactions (i.e., Delphi, simulation, synergy, brainstorming), or correlated through opinion surveys and sampling techniques. Separately or in combination, these methods form the ultimate test of human foresight.

Individuals and groups perform such forecasting in order to expand their horizons into the future. Whether the result of these procedures only confirms some common sense expectation or produces a surprising scenario, the forecasting process forces the human mind to transcend the ordinary and reach beyond into the unthinkable. In this way, it opens new alternatives and widens the options for future action.

Because of the number and diversity of opinions about the future, the best way to choose among them is by consensus of those best qualified in these matters. If we can find a consensus or at least majority opinion supporting a particular forecast, then-unless 
there are other indications to the contrary-we must accept it as credible. If nothing else, when many critical people believe something is going to happen, they will act in such way as to bring it about. So the safest bet, assuming there are no other forces to the contrary, is to assign higher probability of realization to consensual forecasts.

Of course, it is better if consensual credibility of what is possible can be verified by policy analysis of what is intended; and better still, validated by mathematical calculation of what is deterministicly probable. Utilizing these related methods of forecasting in the various degrees of applicability in any particular case, one can arrive at the best possible result. Alternatively, if a question or forecast cannot be verified, validated, or believed to any extent, it should be rejected as improbable, unintended, and incredible.

We have thus, one way or another, come to the end of the process as it is illustrated in the flowchart (Fig. 1). This, along with the elaboration we just completed and the summary of the matrix (Table 1), should have by now clarified the structure and function of this model procedure. At this stage, when the initial construction has only been achieved, it would be better to forego any further details; so as to allow its practical application enough flexibility to evolve appropriate forms for specific contents.

\section{Conclusion}

Once we accept the inevitability of forecasting in human affairs, coupled with the varying predictability of future events, it behooves us to strive toward greater sophistication in our forecasts. To that end, this article emphasizes the necessity of more discriminating forecasting based on definite standards of acceptability. Since we cannot avoid making forecasts, explicitly or implicitly, we must be able to access the quality of such forays into the future, so that we can differentiate subjective speculations from objective prognosis and the combinations in between.

What we have brought out in this article is that there are three distinct bases for claiming any knowledge about the future. The first rests on the assumption that the future is a direct continuation of the past; therefore, if we know the past, we can deduct from it the future. In its starkest form, this is the determinism of natural laws that makes the future inexorably follow from the givens of the past in a cause-effect relationship.

The second basis is the opposite of the first, in assuming that the future is made by the present and thus can be known in the present. This position assigns man the free will to create his future; so that he who can plan ahead knows where he is going. The only reason that prevents us from knowing the future in this case is either our inadequate or contradictory planning, which we can correct by integrated social-system plans.

Finally, the third position ignores or denies the previous two and asserts the mystery of the future in general. Only exceptional circumstances or people can reveal glimpses of this mystery. The gift to foresee events or portends from the future is a talent that only a few possess and cannot be explained in ordinary terms.

These three claims correspond to the scientific, humanistic, and prophetic orientations about the nature of things and exhaust the possibilities of forecasting. Therefore, it was on their basis that we constructed our tripartite model; utilizing each one alone and in combination with the others for a definitive framework of forecast assessment. In this way, we tried to cover all eventualities by relating past data, present intentions, and future opinions into a systematic process of trend calculation, policy analysis, and scenario building.

Because of the variable combinations of determinacy and volition in human affairs, as well as the multiplicity of factors influencing the evolution of events, we should not 
expect too much from any forecasting methodology. All one can hope for is an effective mixture of objective and subjective criteria that take advantage of the most appropriate scientific techniques together with the best available human judgment. Only a judicious application of all these elements can optimize our forecasting capabilities.

Therefore, what preceded should be taken with an attitude of caution and flexibility. It is more the spirit than the letter of the model that should be emphasized and applied to reality. Moreover, in order to make the model comprehensive we had to keep it on a highly theoretical and generally abstract level. The tactical modalities and practical logistics still remain to be worked out along with its application to specific case studies. All that, however, could hardly be done here in this short article, which may only become a prolegomenon to a theory of social forecasting.

This paper was written on the basis of the author's research undertaken for the Policy Analysis Group of the Department of External Affairs of Canada. Thanks is hereby given to that department for its support.

\section{Selected Bibliography}

1. Bestuzhev-Lada, I. V., Social Forecasting, Philosophy Publishing House, Moscow, 1969.

2. Enzer, S., Delphi and Cross-Impact Techniques, Institute for the Future, Middletown, Conn., 1970.

3. Jantsch, E. Technological Forecasting in Perspective, OECD, Paris, 1967.

4. Kahn, H., On Studying the Future, Hudson Institute, New York, 1975.

5. Martino, J. P., Technological Forecasting for Decision-Making, Elsevier, New York, 1972.

6. Mau, J. and Bell, W., The Sociology of the Future, Russell Sage, New York, 1971.

7. Meadows, P., The Many Faces of Change, Scheukman, Cambridge, 1971.

8. Phillips, W. and Thorson, S. The Behavioural Sciences in the Long-Range Planning and Policy Forecasting, U.S. Government Report, Washington, D.C., 1976

9. Platt, J. Perception and Change, University of Michigan Press, Ann Arbor, 1970.

10. Polak. F. L., Prognostics, Elsevier, Amsterdam, 1971.

11. Somit, A., Political Science and the Study of the Future, Dryden, Hinsdale, 1974.

12. Tait, J., Ways of Thinking about the Future, Canadian Government, Ottawa, 1975.

13. Theobald, R., Futures Conditional, Bobbs-Merrill, New York, 1972.

14. Young, M., Forecasting and the Social Sciences, Heinemann, London, 1968. 